\title{
Level densities of transitional Sm nuclei
}

\author{
R. Capote \\ Nuclear Data Section, International Atomic Energy Agency, Vienna, Austria
}

\author{
A. Ventura \\ Ente Nuove Tecnologie, Energia e Ambiente, and Istituto Nazionale di Fisica Nucleare, Bologna, Italy \\ F. Cannata \\ Dipartimento di Fisica dell' Università and Istituto Nazionale di Fisica Nucleare, Bologna, Italy
}

J. M. Quesada

Departamento de Física Atomica, Molecular y Nuclear, Universidad de Sevilla, Spain

(Received 11 March 2005; published 28 June 2005)

\begin{abstract}
Experimentally determined level densities of the transitional isotopes ${ }^{148,149,150,152} \mathrm{Sm}$ at excitation energies below and around the neutron binding energy are compared with microcanonical calculations based on a Monte Carlo approach to noncollective level densities, folded with a collective enhancement estimated in the frame of the interacting boson model (IBM). The IBM parameters are adjusted so as to reproduce the low-lying discrete levels of both parities, with the exception of the odd-mass nucleus, ${ }^{149} \mathrm{Sm}$, where complete decoupling of the unpaired neutron from the core is assumed.
\end{abstract}

DOI: 10.1103/PhysRevC.71.064320

PACS number(s): 21.10.Ma, 21.10.Pc, 21.10.Re, 27.70.+q

\section{INTRODUCTION}

Interest in the study of nuclear excitations in the continuum below the neutron binding energy $B_{n}$ has been revived in recent years by a new method of analysis of $\gamma$-ray transitions, which permits simultaneous extraction of the level density at the excitation energy to which the nucleus under study decays and of the $\gamma$ strength function at the difference of the initial and final excitation energies [1]. For rare-earth nuclei, to which the method was applied for the first time [2], both energies are in the continuum region, thus allowing an investigation of various thermodynamical quantities, such as temperature and entropy, versus excitation energy. In particular, for the lanthanides analyzed in Ref. [2], ${ }^{162} \mathrm{Dy},{ }^{166} \mathrm{Er}$, and ${ }^{172} \mathrm{Yb}$, temperature does not increase monotonically with excitation energy, but shows some broad oscillations, interpreted as a consequence of the breaking of nucleon pairs and, at higher energies, of the possible quenching of pair correlations.

Detailed analyses of the experimental results for these nuclei were published in a serie of papers: In particular, total level densities versus excitation energy were obtained

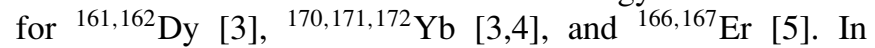
Refs. [3,5], heat capacity versus temperature, deduced from total level density, indicates a phaselike transition from the pair-correlate ground state to an uncorrelated one at a critical temperature $T_{c} \simeq 0.5 \mathrm{MeV}$.

It has been known for years that, in addition to pairing and shell effects, the level densities of deformed nuclei, like the lanthanides analyzed in Refs. [2-5], are strongly enhanced by collective effects, mainly of rotational nature, that are already sizable at excitation energies of the order of $B_{n}$. On the additional assumption of adiabatic decoupling of noncollective and collective degrees of freedom, strictly valid at excitation energies that are much higher than the average collective energy, total level densities are simply obtained by folding noncollective level densities, obtained by a microscopic model, with collective level densities, usually given by some simple quantum model, such as the axially symmetric rotor or the harmonic oscillator, for rotations and vibrations, respectively. At excitation energies of the order of $B_{n}$, rotational effects play the main role: The relevant formalism [6] was applied long ago to the analysis of neutron resonance spacings of nuclei in the lanthanide and actinide regions [7]. Damping of collectivity with increasing energy [8] will be neglected in the low-energy region of interest to the present work.

Recently, the method of Ref. [1] has been applied to weakly deformed nuclei in the same mass region and total level densities and $\gamma$ strength functions have been extracted for ${ }^{148,149} \mathrm{Sm}$ [9]. It is not expected that the collective effects in the level densities of even-even transitional nuclei are adequately reproduced by means of a simple model, like the axially symmetric rotor used for strongly deformed nuclei or the oscillator adopted for spherical nuclei. However, it is known that the low-lying discrete levels and related electromagnetic transitions for this kind of nuclei are well reproduced by the interacting boson model (IBM) [10], an algebraic model of collective excitations with U(6) symmetry.

$\mathrm{Sm}$ isotopes constitute a transitional chain from spherical to axially deformed nuclei, corresponding to the IBM limiting symmetries $\mathrm{U}(5)$ and $\mathrm{SU}(3)$, respectively. By resorting to the coherent state formulation [10] of the IBM, it is possible to interpret the $\mathrm{U}(5) \rightarrow \mathrm{SU}(3)$ shape transition as a quantum phase transition, whose order and critical point can be determined by investigating the behavior of the derivatives of the nuclear ground-state energy with respect to a suitable control parameter. In the Sm chain, the best candidates to be critical are ${ }^{150} \mathrm{Sm}$, on the basis of a recent study that combines 
coherent state formulation with catastrophe theory [11], and ${ }^{152} \mathrm{Sm}$ [12], where selected collective bands of positive parity and related electromagnetic transitions are nicely reproduced in the frame of the $\mathrm{X}(5)$ critical symmetry [13], corresponding to an analytical solution of the Bohr collective Hamiltonian.

Since the discrete spectra of the transitional even-even isotopes ${ }^{148,150,152} \mathrm{Sm}$ show so many interesting features, it appears rather natural to extend the analysis to the continuum. However, the collective level density generated by the IBM cannot be compared directly with experimental data, like those of Ref. [9], which refer to the total level density: The latter can be estimated, for an even-even nucleus, by folding the IBM level density with the density of incoherent quasiparticle excitations, generated by a realistic microscopic model. Since we are also interested in the possible fluctuations of the level density connected with the breaking of Cooper pairs with increasing energy, the calculations will be carried out in the microcanonical ensemble.

The paper is organized as follows: Sec. II describes the collective level density, Sec. III the noncollective level density and Sec. IV the total level density. Finally, Sec. $\mathrm{V}$ is dedicated to the comparison with experimental data and Sec. VI to conclusions and perspectives. A comparison of our formalism with traditional treatments of collective enhancement of nuclear level densities is worked out in detail in the Appendixes.

\section{COLLECTIVE LEVEL DENSITY}

As is known, the IBM commonly used in the analysis of collective excitations of non-magic even-even nuclei is formulated in the laboratory frame, so that diagonalization of the IBM Hamiltonian yields the energies $E_{c}$, of collective levels with good angular momentum $J$ and parity $\pi$. In the present work, we use the simplest version of the model, IBM-1, which does not distinguish between neutron and proton degrees of freedom. The number of proton pairs, $N_{\pi}$, and the number of neutron pairs, $N_{v}$, in the corresponding valence shells define the effective boson number $N_{B}=N_{\pi}+N_{\nu}$, which is a conserved quantity that is the corresponding operator $\widehat{N}$, commutes with the Hamiltonian $\widehat{H}$. Since the bosons of the model simulate collective fermion pairs, the Pauli principle is approximately taken into account by defining the boson number as the number of particle pairs, or of hole pairs, whichever is less, in the given valence shells.

The density of collective levels of given angular momentum and parity is simply written as a sum of Dirac delta functions centered on the collective energies $E_{c}(J, \pi)$ :

$$
\rho_{\text {coll }}(E, J, \pi)=\sum_{c} \delta\left[E-E_{c}\left(J_{c}, \pi_{c}\right)\right] \delta_{J, J_{c}} \delta_{\pi, \pi_{c}},
$$

and the total density of collective levels of given parity is obtained by summing Eq. (1) over $J$ from 0 to the maximum angular momentum $J_{\max }(\pi)$ allowed by the finite boson number $N_{B}$ :

$$
\rho_{\text {coll }}(E, \pi)=\sum_{J=0}^{J_{\max }(\pi)} \sum_{c} \delta\left[E-E_{c}(J, \pi)\right] .
$$

Since a level with angular momentum $J$ corresponds to $2 J+1$ degenerate sublevels, or states, the collective state density is simply

$$
\omega_{\text {coll }}(E, \pi)=\sum_{J=0}^{J_{\max }(\pi)}(2 J+1) \sum_{c} \delta\left[E-E_{c}(J, \pi)\right] .
$$

In the present work, we also need the density of collective states with given projection $M$ on an arbitrary quantization axis:

$$
\begin{aligned}
\omega_{\text {coll }}(E, M, \pi)= & \sum_{J=0}^{J_{\max }(\pi)}(2 J+1) \\
& \times \sum_{c} \delta\left[E-E_{c}(J, \pi)\right] f_{\text {coll }}(M, \pi),
\end{aligned}
$$

where the distribution in $M$ of states of given parity $\pi, f_{\text {coll }}(M, \pi)$, can be normalized to unity in the finite range from $-J_{\max }(\pi)$ to $+J_{\max }(\pi)$ and is directly estimated from the calculated set of collective levels of given parity $\pi$.

Collective levels of positive parity can be obtained by diagonalization of the standard IBM-1 Hamiltonian $\widehat{H}_{s d}$, containing free-boson terms and two-body interactions of $s$ and $d$ bosons, which are the boson images of collective nucleon pairs coupled to angular momentum and parity $L^{\pi}=0^{+}$and $2^{+}$, respectively. The $1+5$ boson degrees of freedom span a dynamical U(6) algebra. Consistent extension of the model to collective states of negative parity and of parity changing electromagnetic transitions requires introduction of $p$ and $f$ bosons, with $L^{\pi}=1^{-}$and $3^{-}$, respectively, corresponding to $3+7$ additional degrees of freedom, so that the dynamical algebra is enlarged to $\mathrm{U}(16)$ [14-17]. However, $p$ bosons play a minor role in reproducing the energies of the low-lying levels of negative parity and are neglected in the present work, where the levels of negative parity are obtained by coupling only one $f$ boson to $s$ and $d$ bosons. Therefore, $J_{\max }(\pi=+)=2 N$ and $J_{\max }(\pi=-)=2 N+1$.

The $s d f$ Hamiltonian adopted in the present work is the same as that of Ref. [18] and can be written in the form

$$
\begin{aligned}
\widehat{H}_{s d f}= & \varepsilon_{d} \widehat{n}_{d}+\varepsilon_{f} \widehat{n}_{f}+\alpha \widehat{P}^{\dagger} s d f \cdot \widehat{P}_{s d f}+\beta \widehat{Q}_{s d f} \cdot \widehat{Q}_{s d f} \\
& +\gamma \widehat{L}_{d f} \cdot \widehat{L}_{d f}+\delta \widehat{Q}_{f} \cdot \widehat{Q}_{s d}+\epsilon \widehat{L}_{d} \cdot \widehat{L}_{f} .
\end{aligned}
$$

Here, $\widehat{n}_{d}$ and $\widehat{n}_{f}$ are the number operators of $d$ and $f$ bosons, respectively, and the multipole operators are defined as follows:

$$
\begin{aligned}
\widehat{P}_{s d f}^{\dagger} & =-s^{\dagger} \cdot s^{\dagger}+d^{\dagger} \cdot d^{\dagger}+f^{\dagger} \cdot f^{\dagger}, \\
\widehat{Q}_{s d} & =\left[s^{\dagger} \times \widetilde{d}+d^{\dagger} \times \widetilde{s}\right]^{(2)}-\frac{\sqrt{7}}{2}\left[d^{\dagger} \times \widetilde{d}\right]^{(2)}, \\
\widehat{Q}_{f} & =-\frac{3}{10} \sqrt{42}\left[f^{\dagger} \times \widetilde{f}\right]^{(2)}, \\
\widehat{Q}_{s d f} & =\widehat{Q}_{s d}+\widehat{Q}_{f}, \\
\widehat{L}_{d} & =\sqrt{10}\left[d^{\dagger} \times \widetilde{d}\right]^{(2)}, \\
\widehat{L}_{f} & =2 \sqrt{7}\left[f^{\dagger} \times \widetilde{f}\right]^{(1)}, \\
\widehat{L}_{d f} & =\widehat{L}_{d}+\widehat{L}_{f} .
\end{aligned}
$$


TABLE I. Boson number $N_{B}$ and coefficients (in $\mathrm{keV}$ ) of the $s d f$ Hamiltonian for $\mathrm{Sm}$ isotopes.

\begin{tabular}{lrccccccc}
\hline \hline Nucleus & $N_{B}$ & $\epsilon_{d}$ & $\epsilon_{f}$ & $\alpha$ & $\beta$ & $\gamma$ & \multicolumn{1}{c}{$\delta$} & $\varepsilon$ \\
\hline${ }^{148} \mathrm{Sm}$ & 8 & 696.5 & 1303.0 & -1.0 & -10.0 & 4.0 & -136.1 & -3.0 \\
${ }^{150} \mathrm{Sm}$ & 9 & 546.5 & 1303.0 & -1.0 & -10.0 & -3.0 & -136.1 & -3.0 \\
${ }^{152} \mathrm{Sm}$ & 10 & 442.3 & 1406.5 & 4.0 & -17.0 & -1.0 & -46.3 & -1.0 \\
\hline \hline
\end{tabular}

In the collective Hamiltonian (5), the monopole-monopole interaction, $\widehat{P}_{s d f}^{\dagger} \cdot \widehat{P}_{s d f}$, the quadrupole-quadrupole interaction, $\widehat{Q}_{s d f} \cdot \widehat{Q}_{s d f}$, and the square of total angular momentum, $\widehat{L}_{d f} \cdot \widehat{L}_{d f}$, modify the standard $s-d$ multipole interactions by including $f$ bosons, whereas the last two cross-multipole terms on the right-hand side of the same equation improve the fit of octupole bands in deformed nuclei. Hamiltonian (5) is thus more general than the one first used in computing negative parity states in Sm isotopes [19].

The eigenvalues of $\widehat{H}_{s d f}$, whose coefficients (in $\mathrm{keV}$ ) are given in Table I, are obtained by numerical diagonalization using a modified version of the Octupole code [20].

The experimental levels of both parities are satisfactorily reproduced up to an excitation energy $E \simeq 1.5 \mathrm{MeV}$ and angular momentum $J_{\max }=10$. To use IBM results in a collective enhancement of intrinsic level densities, collective states have been calculated up to $J=J_{\max }$. The $M$ distributions of collective states turn out to be rather well approximated by Gaussians centered at $M=0$, with characteristic FWHM values of the order of $J_{\max }$.

Figure 1 shows the collective level densities of ${ }^{148,150,152} \mathrm{Sm}$ calculated by IBM for positive and negative parities separately according to Eq. (2). The level densities steadily increase if one goes from the spherical nucleus ${ }^{148} \mathrm{Sm}$ to the deformed nucleus ${ }^{152} \mathrm{Sm}$. It is interesting to remark that the increase is faster for negative parity than for positive parity; therefore the use of $f$ bosons in the IBM is an important requirement for the proper description of the collective enhancement in the Sm isotopic chain. As a consequence, the collective enhancement factor of total level density turns out to be much higher in the deformed case than in the spherical one.

\section{NONCOLLECTIVE LEVEL DENSITY}

Since the collective level density is computed in the laboratory frame, so must be the noncollective one; thus, both collective and noncollective angular momenta are good quantum numbers in the present approach. In particular, singleparticle levels have been generated in a spherical Woods-Saxon potential with parameters appropriate to the Sm region. It is important to remark that the noncollective level density has a weak dependence on deformation, whose main effect is a redistribution of levels, without significant changes in the average density.

Here and in the following, we shall usually refer to the noncollective level density as the intrinsic level density, since this is the traditional name under which it is known in the literature (see, e.g., Ref. [6]). In the present work, intrinsic is equivalent to noncollective and does not imply that calculations are done in an intrinsic reference frame.

Several methods of generating excited multiparticle configurations of an atomic nucleus from given sets of singleparticle states were proposed in the course of years, both in the microcanonical and the grand-canonical frameworks, and are available in the literature. In particular, an exact recursive method of interest to the present work was originally formulated by Williams [21]: It is based on the use of exact recursion relations to expand the grand partition function, but it does not rely on the saddle-point approximation used in the traditional grand-canonical approach. It has the advantage of requiring much shorter computation time than a combinatorial approach, which counts all possible configurations, but cannot deal with residual interactions, at least in its original form.

In the combinatorial methods, such as the odometer approach of Hillman and Grover [22], level densities are

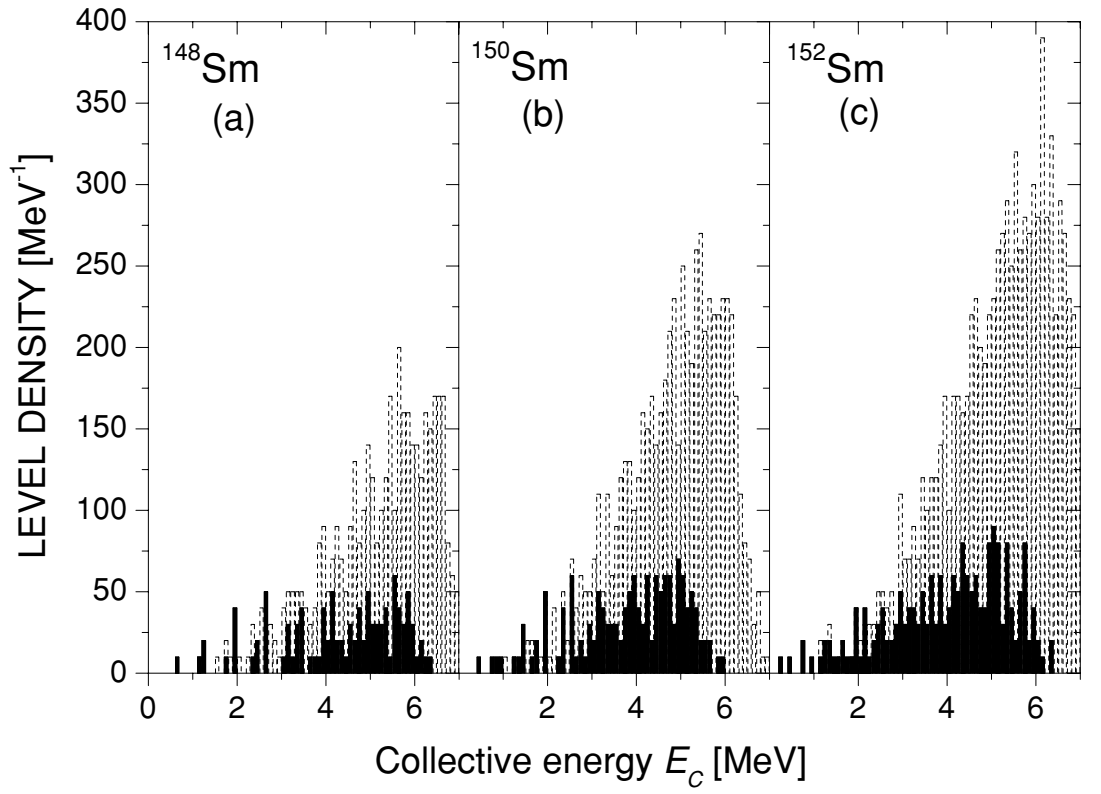

064320-3
FIG. 1. Collective level densities of ${ }^{148,150,152} \mathrm{Sm}$ vs excitation energy. Solid histograms: positive parity; dashed histograms: negative parity. 
computed numerically by performing an exhaustive counting of excited configurations. More recently, combinatorial calculations were carried out by means of the Densidad code [23], based on a model proposed by Herman and Reffo [24-26], to estimate level densities with a fixed number of particles and holes (excitons), for use in pre-equilibrium models of nuclear reactions. In the frame of an independent particle picture of atomic nuclei, this method is exact but very time consuming and becomes intractable at high excitation energies or for large shell model spaces (i.e., for medium and heavy nuclei). A residual pairing interaction can be included in the usual Bardeen-Cooper-Schrieffer (BCS) approximation, at the cost of greatly increased computer time.

It becomes thus natural to resort to a Monte Carlo (MC) technique, like the one proposed by Cerf [27], to avoid exhaustive counting of excited configurations and reduce computer time, without sizable loss of accuracy. In general, $\mathrm{MC}$ algorithms are known to be very efficient in solving combinatorial problems. In this work the MC procedure is followed to extract a random sample of excited states in the considered energy range. This very small fraction of configuration space is assumed to be representative of the whole ensemble. Since the level density increases exponentially, it is difficult to do a MC sampling uniformly over the entire energy range of interest. In what follows we make use of the Metropolis sampling scheme [28] to achieve uniform sampling, as explained in detail by Cerf [27]. A MC estimator for the intrinsic state density of the $N$-neutron or $Z$-proton system with given angular momentum projection $M$ and parity $\pi$ is given by

$$
\omega_{\text {intr }}^{N, Z}(E, M, \pi) \simeq s \sum_{j=1}^{L} \frac{\delta\left(E-E_{C_{j}}\right) \delta_{M, M_{j}} \delta_{\pi, \pi_{j}}}{W\left(C_{j}\right)},
$$

where $C_{j}$ are random single-particle configurations along a long enough random walk of length $L$, and $W\left(C_{j}\right)$ are the weight functions associated with each configuration. The scale factor $s$, which is needed to normalize the state density, is derived from an exact recursive calculation by means of the Totstade code [29], where the recursive method of Williams [21] is implemented. The recursive method, however, cannot yield spin-parity distributions, nor can it take into account a residual pairing interaction: Therefore, we just need it to normalize the sampled MC state density. The calculated recursive state density $\omega_{\text {intr }}^{\text {rec }}(E)$ is also used as the weight function $W\left(C_{j}\right)=W\left(E_{C_{j}}\right)$ of the Metropolis sampling scheme, where $E_{C_{j}}$ is the energy of a given $j$ th configuration [27]. To derive the normalization factor needed in Eq. (13), the total number of states, $N_{\text {rec }}$, is calculated up to the maximum excitation energy $E_{\max }$ :

$$
N_{\mathrm{rec}}=\int_{0}^{E_{\max }} \omega_{\text {intr }}^{\mathrm{rec}}(E) d E .
$$

Using the normalization condition

$$
N_{\mathrm{rec}}^{N, Z}=\int_{0}^{E_{\max }} d E \sum_{M} \sum_{\pi} \omega_{\mathrm{intr}}^{N, Z}(E, M, \pi)
$$

and Eqs. (13)-(15) we obtain the following expression of the scale factor $s$ :

$$
s=\frac{N_{\mathrm{rec}}^{N, Z}}{\sum_{j=1}^{N} \frac{1}{W\left(C_{j}\right)}} .
$$

Residual pairing interactions treated in the BCS approximation are easily introduced in the MC formalism following again the approximate method of Ref. [27]: For each sampled excited configuration $C_{j}$, the usual BCS equations for energy gap and particle number are solved by blocking the orbits occupied by unpaired nucleons, and the computed pairing energy $P_{C_{j}}$ is used to correct the energy $E_{C_{j}}$ of the sampled configuration, replaced by $E_{C_{j}}-P_{C_{j}}$ in the computation of the MC state density, Eq. (13), which is changed into

$$
\omega_{\text {intr }}^{\mathrm{BCS}}(E, M, \pi) \simeq s \sum_{j=1}^{N} \frac{\delta\left(E-E_{C_{j}}+P_{C_{j}}\right) \delta_{M, M_{j}} \delta_{\pi, \pi_{j}}}{W\left(E_{C_{j}}\right)},
$$

because of the pairing energy correction $P_{C_{j}}$. The energy $E_{C_{j}}$ used to calculate the weight function does not contain the pairing correction, therefore the state density without pairing can be computed at the same time, so the scaling factor $s$ is common to both formulations, with pairing and without it. In this approximate treatment of pairing, the excitations involving promoted pairs are neglected, but this helps to minimize the overlap with the collective excitations computed in the IBM, since the interacting bosons of the model simulate collective fermion pairs and the eigenstates of the IBM Hamiltonian can be considered as excitations resulting from promoted collective pairs.

The intrinsic state density $\omega_{\text {intr }}(E, M, \pi)$ of the whole nucleus with given angular momentum projection $M$ and parity $\pi$ is obtained by separate calculations of neutron and proton state densities, $\omega_{\text {intr }}^{N}(E, M, \pi)$ and $\omega_{\text {intr }}^{Z}(E, M, \pi)$, respectively, by means of Eq. (13), and their subsequent convolution is performed according to the formula for the coefficients of the Cauchy product of two infinite series

$$
\begin{aligned}
\omega_{\text {intr }}(E, M, \pi)= & \sum_{Q=0}^{E} \sum_{\pi_{N}} \sum_{\pi_{Z}} \sum_{M_{N}} \sum_{M_{Z}} \omega_{\text {intr }}^{N}\left(Q, M_{N}, \pi_{N}\right) \omega_{\text {intr }}^{Z} \\
& \times\left(E-Q, M_{Z}, \pi_{Z}\right) \delta_{\pi, \pi_{N} \pi_{Z}} \delta_{M, M_{N}+M_{Z}} .
\end{aligned}
$$

\section{TOTAL LEVEL DENSITY}

On the assumption of complete decoupling of intrinsic and collective degrees of freedom, at low energy $E$, where both collective and intrinsic levels are discrete, the total density of states of given spin projection $M$ and parity $\pi$ can be put in the form

$$
\begin{aligned}
\omega(E, M, \pi)= & \sum_{\pi_{i}} \sum_{\pi_{c}} \sum_{c=0}^{N_{c}\left(\pi_{c}\right)} \sum_{i=0}^{N_{i}\left(\pi_{i}\right)} \sum_{M_{i}} \sum_{M_{c}} \delta\left[E-E_{c}\left(M_{c}, \pi_{c}\right)\right. \\
& \left.-\epsilon_{i}\left(M_{i}, \pi_{i}\right)\right] \delta_{M_{c}+M_{i}, M} \delta_{\pi_{c} \pi_{i}, \pi},
\end{aligned}
$$

where $E_{c}, M_{c}, \pi_{c}$ are the collective and $\epsilon_{i}, M_{i}, \pi_{i}$ are the intrinsic excitation energies, spin projection and parities. The 
ground-state collective and intrinsic energies, $E_{0}$ and $\epsilon_{0}$, respectively, are both set to zero. According to formula (19), in particular, at $E<\epsilon_{1}$, the lowest two-quasiparticle excitation, the total level density of an even-even nucleus coincides with the collective one.

At excitation energy $E$ much greater than $\left\langle E_{c}\right\rangle$, the average collective energy, where the intrinsic state density can be well approximated by a continuum, it is more convenient to start from the folding of the intrinsic and collective state densities at given projection of total angular momentum $M=M_{i}+M_{c}$ :

$$
\begin{aligned}
\omega(E, M, \pi)= & \sum_{\pi_{i}} \sum_{\pi_{c}} \sum_{c=0}^{N_{c}\left(\pi_{c}\right)} \int_{0}^{\infty} d E_{i} \sum_{M_{i}+M_{c}=M} \omega_{\text {intr }}\left(E_{i}, M_{i} \pi_{i}\right) \\
& \times \omega_{\text {coll }}\left(E-E_{i}, M_{c}, \pi_{c}\right) \delta_{\pi_{c} \pi_{i}, \pi},
\end{aligned}
$$

which immediately reduces to formula (19) if the intrinsic and collective state densities are discretized again as

$$
\begin{aligned}
\omega_{\text {intr }}\left(E_{i}, M_{i}, \pi_{i}\right)= & \sum_{j, M_{j}, \pi_{j}} \delta\left[E_{i}-\epsilon_{j}\left(M_{j}, \pi_{j}\right)\right] \\
& \times \delta_{\pi_{i}, \pi_{j}} \delta_{M_{i}, M_{j}} \\
\omega_{\mathrm{coll}}\left(E-E_{i}, M_{c}, \pi_{c}\right)= & \sum_{l, M_{l}, \pi_{l}} \delta\left[E-E_{i}-E_{l}\left(M_{l}, \pi_{l}\right)\right] \\
& \times \delta_{\pi_{c}, \pi_{l}} \delta_{M_{c}, M_{l}} .
\end{aligned}
$$

The observable level density of given spin $J$ and parity $\pi, \rho(E, J, \pi)$, is obtained from the calculated state density $\omega(E, M, \pi)$ of the whole nucleus, given by Eq. (19), using a formula valid in the case of spherical symmetry (see, e.g., Ref. [30]),

$$
\rho(E, J, \pi)=\omega(E, M=J, \pi)-\omega(E, M=J+1, \pi) .
$$

The total level density is finally obtained by summing this expression over $J$ and $\pi$,

$$
\rho(E)=\sum_{J \pi} \rho(E, J, \pi)
$$

All level density calculations carried out in the present work make direct use of formulas (19)-(24). However, comparison of our formalism with the traditional treatment of collective enhancement of deformed nuclei [7], albeit not necessary to the present work, allows us to gain a qualitatively interesting physical insight. To this aim, a more compact approximation to formula (20) is worked out in Appendix A and this is compared with the corresponding formalism for axially symmetric rotors in Appendix B.

\section{NUMERICAL RESULTS}

The hybrid formalism described in the preceding sections, where an intrinsic state density from MC calculations done in the microcanonical ensemble is folded with a collective state density computed by means of the interacting boson model, on the assumption of complete decoupling of intrinsic and collective degrees of freedom, is applied to four transitional Sm isotopes. The resulting total level density $\rho(E)$ is plotted in Fig. 2 as a function of $E$ up to $E=B_{n}$, the neutron binding energy, and compared with experimental data in the energy range from $1 \mathrm{MeV}$ to $B_{n}-1 \mathrm{MeV}$ for ${ }^{148,149} \mathrm{Sm}$ [9].

In the case of ${ }^{149} \mathrm{Sm}$ we have folded the intrinsic state density of the odd-mass nucleus with the collective state density of its even-even core, ${ }^{148} \mathrm{Sm}$, because we have assumed complete decoupling of the odd neutron from the core. Not surprisingly, this approximation is particularly poor at low excitation energies, where the leading contribution to the total level density is the collective one. At such energies, the collective level density could be computed in the interacting boson-fermion model (IBFM) [31], where the coupling of the odd fermion with the even-even core of an odd-mass nucleus is properly taken into account. This kind of calculation, however, lies beyond the scope of the present work, because it would require additional work in its own, namely, the extension of the standard IBFM, where the odd fermion is coupled with $s$ and $d$ bosons only, to the coupling with negative-parity bosons.

Other important pieces of information about level densities at the neutron binding energy are the average spacings of $s$-wave and $p$-wave resonances detected in neutron capture by a target nucleus with one less neutron than the nucleus under consideration. A recent comprehensive compilation of neutron resonance spacings is included in the Reference Input Parameter Library, Version 2 (RIPL-2) [32], which contains recommended values for $296 s$-wave resonance spacings and $82 p$-wave resonance spacings. Table II compares the values of the model-independent $s$-wave resonance spacings $D_{0}$ computed in the present approach by counting relevant resonances for the four Sm compound nuclei under consideration with the recommended values of the RIPL-2 compilation. By definition

$$
D_{0}= \begin{cases}{\left[\rho\left(B_{n}+\Delta E / 2,1 / 2^{+}\right)\right]^{-1},} & I_{t}=0^{+} \\ \left\{\rho\left[B_{n}+\Delta E / 2,\left(I_{t}^{\pi}-1 / 2\right)^{\pi}\right]+\rho\left[B_{n}+\Delta E / 2,\left(I_{t}^{\pi}+1 / 2\right)^{\pi}\right]\right\}^{-1}, & I_{t} \neq 0^{+}\end{cases}
$$

where $I_{t}^{\pi}$ is the ground-state spin parity of the target nucleus and $\Delta E$ is the energy interval for counting the resonances.

In the case of ${ }^{152} \mathrm{Sm}$, there is also an experimental value of $D_{0}$ recently obtained by the $\mathrm{n}_{-} \mathrm{TOF}$ collaboration [33] in neutron capture by ${ }^{151} \mathrm{Sm}$, which is slightly higher than the RIPL-2 recommendation and our theoretical result. The agreement between theory and experiment is satisfactory in the case of ${ }^{148,152} \mathrm{Sm}$ and underestimates the experimental values by a factor of 2 in the case of ${ }^{149,150} \mathrm{Sm}$. However, it is to be stressed again that our theoretical values are obtained on the crude assumption of decoupling of intrinsic and collective degrees of freedom, which is poor, in particular, for the 


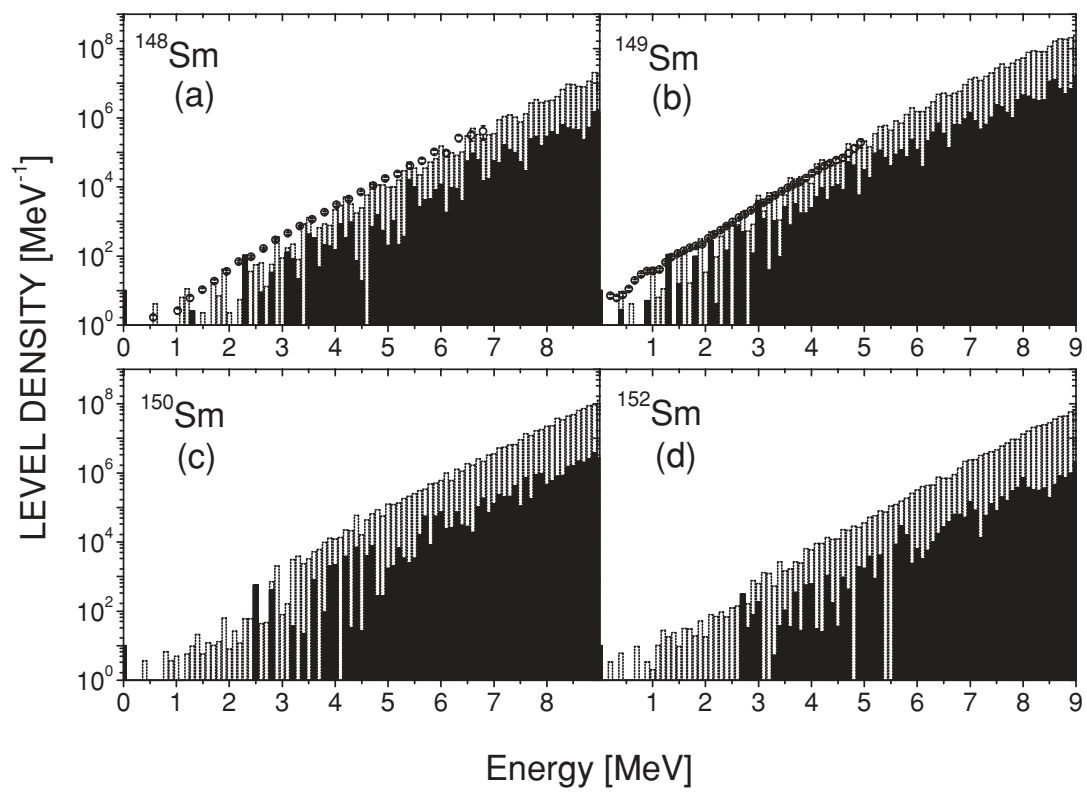

FIG. 2. Total level densities of $148,149,150,152 \mathrm{Sm}$ vs excitation energy (dashed histograms) and corresponding intrinsic level densities (solid histograms). The experimental data (empty circles) of ${ }^{148,149} \mathrm{Sm}$ are taken from Ref. [9].

odd-mass nucleus ${ }^{149} \mathrm{Sm}$. Therefore, we consider the present results as an encouraging approximation; in any case, they emphasize the crucial role played by collective enhancement in level densities around $E \simeq B_{n}$. As can be seen from Fig. 2, without folding with the collective contribution, the noncollective values of $D_{0}$ would overestimate the experimental ones by a factor of 10-50 in this mass region (see also Ref. [34]).

The main advantage of our treatment of collective effects is that the model can be equally well applied to spherical $\left({ }^{148} \mathrm{Sm}\right)$, transitional $\left({ }^{150} \mathrm{Sm}\right)$, and deformed nuclei $\left({ }^{152} \mathrm{Sm}\right)$, whereas the model of Refs. [6,7], briefly described in Appendix B, is valid only in the last case and, in fact, it has already been applied to heavier deformed lanthanides measured by the Oslo group, such as ${ }^{161,162} \mathrm{Dy},{ }^{166} \mathrm{Er}$, and ${ }^{171,172} \mathrm{Yb}[35,36]$.

\section{CONCLUSIONS AND PERSPECTIVES}

The numerical results discussed in Sec. V appear to us, in spite of some simplifying model assumptions, significant enough to encourage improvement of the model in two basic steps: (i) extension to higher excitation energies; (ii) introduction of a proper coupling of intrinsic and collective degrees of freedom for odd nuclei.

TABLE II. $s$-wave resonance spacings for Sm isotopes.

\begin{tabular}{lcccc}
\hline \hline $\begin{array}{l}\text { Compound } \\
\text { Nucleus }\end{array}$ & $B_{n}(\mathrm{MeV})$ & $I_{t}^{\pi}$ & $D_{0 \text { exp. }}(\mathrm{eV})$ & $D_{\text {0calc. }}(\mathrm{eV})$ \\
\hline${ }^{148} \mathrm{Sm}$ & 8.141 & $7 / 2^{-}$ & $(5.1 \pm 0.5)^{\mathrm{a}}$ & $5.4 \pm 0.3$ \\
${ }^{149} \mathrm{Sm}$ & 5.871 & $0^{+}$ & $(100.0 \pm 20.0)^{\mathrm{a}}$ & $53.0 \pm 2.0$ \\
${ }^{150} \mathrm{Sm}$ & 7.985 & $7 / 2^{-}$ & $(2.1 \pm 0.3)^{\mathrm{a}}$ & $0.94 \pm 0.03$ \\
${ }^{152} \mathrm{Sm}$ & 8.257 & $5 / 2^{-}$ & $(1.04 \pm 0.15)^{\mathrm{a}}$ & $1.2 \pm 0.1$ \\
& & & $(1.48 \pm 0.04)^{\mathrm{b}}$ & \\
\hline
\end{tabular}

\footnotetext{
${ }^{\mathrm{a}}$ From Ref. [32].
}

${ }^{\mathrm{b}}$ From Ref. [33].
Step (i) is relatively simple, if one replaces the microcanonical description adopted in the present work with a grand-canonical formalism, at the cost of smoothing out the level density fluctuations related to shell and pairing effects, which are less and less observable with increasing excitation energy. Under this assumption, one could compute the intrinsic state density $\omega_{\text {intr }}(E, M, \pi)$ in the grand-canonical ensemble (see, e.g., Ref. [30]) and then make use of Eq. (A11) of Appendix A for the total state density $\omega(E, M, \pi)$, and derive total level densities $\rho(E, J, \pi)$ by means of Eq. (23).

The problem still to be solved in this approach is the damping of collective effects with increasing temperature, that is, the temperature dependence of the collective partition function $Z_{\text {coll }}\left(T, \pi_{c}\right)$ defined by Eq. (A6) of Appendix A, which is expected to go through a maximum at some finite temperature and then decrease to unity in the $T \rightarrow \infty$ limit. The problem was already faced in Refs. [18,37] under the simplifying assumption that only the effective boson number $N_{B}$ changes with temperature and goes to zero when $T$ goes to infinity. Even in that case, however, a realistic microscopic calculation of $N_{B}(T)$ is needed, since it was recently noticed [38] that the simple BCS calculations at finite temperature done in Refs. [18,37] do not properly deal with quadrupole and octupole bosons, inducing, as a consequence, the transition from a deformed ground state to a spherical equilibrium shape at much lower temperature than the one predicted by selfconsistent mean-field calculations. In addition, the temperature dependence of the IBM Hamiltonian parameters, in particular the $d$ - and $f$-boson energies, might be non-negligible over a broad temperature range, so that a consistent microscopic model of both boson number and Hamiltonian appears to be mandatory.

Once step (i) is taken and computed level densities at high energy compare reasonably well with available experimental data, one might try step (ii), that is, inclusion of the coupling of intrinsic and collective degrees of freedom in the formalism, of particular importance to the treatment of odd-mass and odd-odd nuclei at low excitation energies. 
The most viable computational approach in this case is the simultaneous MC treatment of both mean-field and residual interactions. A finite temperature version of the MC treatment of the intrinsic level density described in Sec. III was proposed by Cerf in Ref. [39] and applied there to the pairing interaction in a single $j$ shell, to check numerical results with the exact analytical results of the seniority model. Since the calculation of thermodynamic quantities is done there in the canonical ensemble, and promoted pairs are treated like bosons, with an approximate inclusion of the Pauli principle, the extension to the boson interactions of the IBM might be feasible, but how to treat boson-fermion couplings is an open matter for investigation.

A completely different approach is the shell model MC (SMMC) method [40,41], where correlation effects are taken into account by including fluctuations of the mean field through the Hubbard-Stratonovich transformation, which permits decomposition of the many body operator $\exp (-\beta H)$, considered as an imaginary-time propagator, in a set of one-body propagators of noninteracting particles moving in fluctuating auxiliary fields. The SMMC method has been extensively applied by Alhassid and coworkers [42] to the calculation of level densities of nuclei in the mass and energy range that can be described in a model space limited to the fpg $_{9 / 2}$ major shell (i.e., nuclei in the $50<A<70$ region) up to excitation energies of the order of $30 \mathrm{MeV}$, by using a residual fermion interaction made of an isovector pairing interaction and a number of isoscalar multipole-multipole interactions, which are all attractive, thus yielding a good-sign Hamiltonian, i.e., avoiding fluctuating signs of integrands in MC integrals over auxiliary fields. Recently, the method has been extended to much higher excitation energies, of the order of $90 \mathrm{MeV}$ for nuclei in the same mass region [43], including also the contribution of scattering states, in addition to the bound states, to the nuclear partition function, which becomes significant for weakly bound nuclei in the temperature range from 2 to $4 \mathrm{MeV}$. At higher temperature the approximation of fixed mean field used in the SMMC calculations loses its meaning. However, it is not trivial how the method could be extended to medium-heavy nuclei $(70<A<240)$, where the IBM and IBFM work at their best in reproducing discrete levels and related electromagnetic transitions.

\section{ACKNOWLEDGMENTS}

It is a pleasure to thank M. Bruno and M. D'Agostino for useful discussions. One of us (R. C.) acknowledges partial financial support from the Physics Department of the University of Bologna and the Bologna Section of the Italian National Institute for Nuclear Physics during his stay in Bologna in 2003.

\section{APPENDIX A: HIGH-ENERGY APPROXIMATION}

If $E \gg\left\langle E_{c}\right\rangle$, the average collective energy, we start from the convolution (20), where we introduce expression (4) for

$$
\begin{aligned}
\omega_{\mathrm{coll}}\left(E_{c}, M_{c}, \pi_{c}\right): & \\
\omega(E, M, \pi)= & \sum_{\pi_{i}} \sum_{\pi_{c}} \int_{0}^{\infty} d E_{i} \sum_{M_{i}+M_{c}=M} \omega_{\mathrm{intr}}\left(E_{i}, M_{i, \pi_{i}}\right) \\
& \times \sum_{I, c}(2 I+1) \delta\left[E-E_{i}-E_{c}\left(I, \pi_{c}\right)\right] \\
& \times f_{\mathrm{coll}}\left(M_{c}, \pi_{c}\right) \delta_{\pi_{c} \pi_{i}, \pi} \\
= & \sum_{\pi_{i} \pi_{c}} \sum_{M_{i}+M_{c}=M} \sum_{I, c}(2 I+1) \omega_{\mathrm{intr}} \\
& {\left[E-E_{c}\left(I, \pi_{c}\right), M_{i}, \pi_{i}\right] f_{\mathrm{coll}}\left(M_{c}, \pi_{c}\right) \delta_{\pi_{i} \pi_{c}, \pi} . }
\end{aligned}
$$

We now assume that the intrinsic state density $\omega_{\text {intr }}\left(E_{i}, M_{i}, \pi_{i}\right)$ also factorizes,

$$
\omega_{\text {intr }}\left(E_{i}, M_{i}, \pi_{i}\right)=\omega_{\text {intr }}\left(E_{i}, \pi_{i}\right) f_{\text {intr }}\left(M_{i}, \pi_{i}\right),
$$

and expand $\omega_{\text {intr }}\left(E-E_{c}, \pi_{i}\right)$ in a Taylor series to first order in the collective energy $E_{c}$, since we have assumed that $E \gg E_{c}$. Therefore

$$
\begin{aligned}
\omega_{\text {intr }}\left[E-E_{c}\left(I, \pi_{c}\right), \pi_{i}\right] \simeq & \omega_{\text {intr }}\left(E, \pi_{i}\right)-E_{c}\left(I, \pi_{c}\right) \\
& \times \frac{\partial}{\partial E} \omega_{\text {intr }}\left(E, \pi_{i}\right) \\
= & \omega_{\text {intr }}\left(E, \pi_{i}\right)\left(1-\frac{E_{c}\left(I, \pi_{c}\right)}{T(E)}\right) \\
\simeq & \omega_{\text {intr }}\left(E, \pi_{i}\right) \exp \left(-\frac{E_{c}\left(I, \pi_{c}\right)}{T(E)}\right) .
\end{aligned}
$$

Here, the inverse temperature corresponding to excitation energy $E$ is, by definition,

$$
\frac{1}{T}=\frac{1}{\omega_{\text {intr }}\left(E, \pi_{i}\right)} \frac{\partial}{\partial E}\left[\omega_{\text {intr }}\left(E, \pi_{i}\right)\right] .
$$

Therefore, Eq. (A1) is transformed into

$$
\begin{aligned}
\omega(E, M, \pi)= & \sum_{\pi_{i} \pi_{c}} \sum_{M_{i} M_{c}} \omega_{\text {intr }}\left(E, \pi_{i}\right) f_{\text {intr }}\left(M_{i}, \pi_{i}\right) f_{\text {coll }}\left(M_{c}, \pi_{c}\right) \\
& \times Z_{\text {coll }}\left[T(E), \pi_{c}\right] \delta_{\pi_{i} \pi_{c}, \pi} \delta_{M_{i}+M_{c}, M},
\end{aligned}
$$

where the colllective enhancement factor is

$$
Z_{\mathrm{coll}}\left(T(E), \pi_{c}\right)=\sum_{I=0}^{I_{\max }\left(\pi_{c}\right)} \sum_{c}(2 I+1) \exp \left(-\frac{E_{c}\left(I, \pi_{c}\right)}{T(E)}\right),
$$

which has the form of a canonical partition function.

Formula (A6) lends itself to the introduction of collectivity damping with increasing temperature, provided the collective model is modified by a temperature dependence of the Hamiltonian parameters. In particular, the behavior of the partition function (A6) on the assumption that only the effective boson number decreases with increasing temperature was first studied at the limiting symmetries of IBM-1 for positive parity only [37], and, more recently, for both parities in the same transitional Sm chain [18] considered in the present work. 
We now make another assumption, that both the intrinsic and collective $M$ distributions are well approximated by Gaussians,

$$
f_{g}\left(M_{g}, \pi_{g}\right)=\frac{1}{\sqrt{2 \pi} \sigma_{g}} \exp \left(-\frac{M_{g}^{2}}{2 \sigma_{g}^{2}}\right),
$$

where index $g$ means either intrinsic $(i)$ or collective $(c)$. It is to be stressed that, in general, the width $\sigma_{g}$ is a function of parity $\pi_{g}$. On these conditions the folding of $M$ distributions can be evaluated analytically:

$$
\begin{aligned}
f\left(M, \pi_{i}, \pi_{c}\right)= & \sum_{M_{i}+M_{c}=M} f_{i}\left(M_{i}, \pi_{i}\right) f_{c}\left(M_{c}, \pi_{c}\right) \\
= & \sum_{M_{i} M_{c}} f_{i}\left(M_{i}, \pi_{i}\right) f_{c}\left(M_{c}, \pi_{c}\right) \delta\left(M-M_{i}-M_{c}\right) \\
\simeq & \int_{-\infty}^{+\infty} d M_{c} f_{i}\left(M-M_{c}, \pi_{i}\right) f_{c}\left(M_{c}, \pi_{c}\right) \\
= & \frac{1}{\sqrt{2 \pi} \sigma_{i}} \frac{1}{\sqrt{2 \pi} \sigma_{c}} \int_{-\infty}^{+\infty} d M_{c} \exp \left(-\frac{M_{c}^{2}}{2 \sigma_{c}^{2}}\right) \\
& \times \exp \left(-\frac{\left(M-M_{c}\right)^{2}}{2 \sigma_{i}^{2}}\right) \\
= & \frac{1}{\sqrt{2 \pi} \sigma_{i}} \frac{1}{\sqrt{2 \pi} \sigma_{c}} \exp \left(-\frac{M^{2}}{2 \sigma_{i}^{2}}\right) \int_{-\infty}^{+\infty} d M_{c} \\
& \times \exp \left(-b M_{c}^{2}+a M_{c}\right),
\end{aligned}
$$

where $a=M / \sigma_{i}^{2}$ and $b=1 / 2\left(\sigma_{i}^{2}+\sigma_{c}^{2}\right) / \sigma_{i}^{2} \sigma_{c}^{2}$. The integral on the right-hand side is elementary, simplifying to

$$
\int_{-\infty}^{+\infty} d M_{c} \exp \left(-b M_{c}^{2}+a M_{c}\right)=\sqrt{\frac{\pi}{b}} \exp \left(\frac{a^{2}}{4 b}\right)
$$

and the final results for $f$ is

$$
f\left(M, \pi_{i}, \pi_{c}\right)=\frac{1}{\sqrt{2 \pi} \sqrt{\sigma_{i}^{2}+\sigma_{c}^{2}}} \exp \left[-\frac{M^{2}}{2\left(\sigma_{i}^{2}+\sigma_{c}^{2}\right)}\right] .
$$

Therefore,

$$
\begin{aligned}
\omega(E, M, \pi)= & \sum_{\pi_{i} \pi_{c}} f\left(M, \pi_{i}, \pi_{c}\right) Z_{\mathrm{coll}}\left[T(E), \pi_{c}\right] \\
& \times \omega_{\mathrm{intr}}\left(E, \pi_{i}\right) \delta_{\pi_{i} \pi_{c}, \pi} .
\end{aligned}
$$

To evaluate the level density at given angular momentum and parity $\rho(E, J, \pi)$, we make use of Eq. (23), where the difference on the right-hand side can be further approximated by a derivative, in the limit of large $J$ :

$$
\begin{aligned}
\rho(E, J, \pi) \simeq & -\left.\frac{\partial}{\partial M} \omega(E, M, \pi)\right|_{M=J+\frac{1}{2}} \\
= & \sum_{\pi_{i} \pi_{c}} \frac{2 J+1}{2 \sqrt{2 \pi}\left(\sigma_{i}^{2}+\sigma_{c}^{2}\right)^{\frac{3}{2}}} \exp \left[-\frac{\left(J+\frac{1}{2}\right)^{2}}{2\left(\sigma_{i}^{2}+\sigma_{c}^{2}\right)}\right] \\
& \times Z_{\text {coll }}\left[T(E), \pi_{c}\right] \omega_{\text {intr }}\left(E, \pi_{i}\right) \delta_{\pi_{i} \pi_{c}, \pi} .
\end{aligned}
$$

The standard expression of the intrinsic level density of spherical nuclei (see, e.g., Ref. [30]) is recovered from this formula when $\pi_{c}=+, \sigma_{c}=0$, and $Z_{\text {coll }} \equiv 1 ; \sigma_{i}$ is identified with the parallel spin cutoff factor $\sigma_{\|}$.
A widely used expression of $\rho(E, J, \pi)$ for deformed nuclei (see, e.g., Ref. [44]) neglects the folding of intrinsic and collective $M$ distributions ( $\left.\sigma_{c}=0\right)$ and uses, instead of $Z_{\text {coll }}$, a collective enhancement factor $K_{\text {rot }} K_{\text {vib }}$, where, differently from our $Z_{\text {coll }}$, rotational and vibrational degrees of freedom are decoupled. These approximations lead to a significant overestimate of collective enhancement, at least at low energy, where collectivity damping with increasing excitation energy can be neglected.

\section{APPENDIX B: COMPARISON OF IBM AND ROTATIONAL ENHANCEMENT FACTORS}

In the traditional treatment of rotational enhancement [6,7] of level densities of axially symmetric nuclei, the intrinsic excitations are labeled with the projection $K$ of total angular momentum on the nuclear symmetry axis, and the corresponding state density $\omega_{\text {intr }}\left(E_{\text {intr }}, K, \pi\right)$ is folded with a simple rotational level density to give the following level density for specific angular momentum $J$ :

$$
\rho(E, J, \pi)=\frac{1}{2} \sum_{K=-J}^{J} \omega_{\text {intr }}\left[E-E_{\text {rot }}(K, J), K, \pi\right],
$$

where

$$
E_{\text {rot }}(K, J)=\frac{\hbar^{2}}{2 \mathfrak{I}_{\perp}}\left[J(J+1)-K^{2}\right],
$$

and $\mathfrak{\Im}_{\perp}$ is the moment of inertia about an axis perpendicular to the nuclear symmetry axis. In the expression of $\rho(E, J, \pi)$, valid for $E>E_{\text {rot }}(K, J)$, the factor of $1 / 2$ accounts for the fact that, when $K \neq 0$, the intrinsic states with projection $\pm K$ combine to form a single band with $J=|K|,|K|+1$, etc., whereas, for $K=0$, only one half of the bands contribute, for a given $J$.

If there are many independent intrinsic degrees of freedom, $\omega_{\text {intr }}(E, K, \pi)$ can be assumed to be normal in $K$ :

$\omega_{\text {intr }}\left(E_{\text {intr }}, K, \pi\right)=\frac{1}{\sqrt{2 \pi} \sigma_{\|}} \exp \left(-\frac{K^{2}}{2 \sigma_{\|}^{2}}\right) \omega_{\text {intr }}(E, \pi)$

where $\sigma_{\|}$is the parallel spin cutoff factor. By repeating for $\rho_{\text {tot }}(E, J, \pi)$ the steps of Taylor expansion to first order in $E_{\text {rot }}$ and introducing of temperature $T$, one obtains the well-known expression

$$
\begin{aligned}
\rho(E, J, \pi)= & \frac{1}{2 \sqrt{2 \pi} \sigma_{\|}} \omega_{\text {intr }}(E, \pi) \\
& \times \sum_{K=-J}^{J} \exp \left[-\frac{J(J+1)}{2 \sigma_{\perp}^{2}}-\frac{K^{2}}{2}\left(\frac{1}{\sigma_{\|}^{2}}-\frac{1}{\sigma_{\perp}^{2}}\right)\right],
\end{aligned}
$$

where

$$
\sigma_{\perp}^{2}=\frac{\Im_{\perp} T}{\hbar^{2}}
$$

is the perpendicular spin-cutoff factor. The total level density $\rho(E, \pi)$ is obtained by summing Eq. (B4) over $J$ :

$$
\rho(E, \pi)=\sum_{J=0}^{\infty} \rho(E, J, \pi) .
$$


Formula (B4) is considerably simplified when the term containing $K^{2}$ is negligible with respect to the term containing $J(J+1)$ in the argument of the exponential. In that case

$$
\begin{aligned}
\rho(E, J, \pi) & \simeq \frac{\omega_{\text {intr }}(E, \pi)}{2 \sqrt{2 \pi} \sigma_{\|}}(2 J+1) \exp \left(-\frac{J(J+1)}{2 \sigma_{\perp}^{2}}\right), \\
\rho(E, \pi) & \simeq \frac{\omega_{\text {intr }}(E, \pi)}{2 \sqrt{2 \pi} \sigma_{\|}} \sum_{J=0}^{\infty}(2 J+1) \exp \left(-\frac{J(J+1)}{2 \sigma_{\perp}^{2}}\right) \\
& \simeq \frac{\omega_{\text {intr }}(E, \pi)}{\sqrt{2 \pi} \sigma_{\|}} \sigma_{\perp}^{2} .
\end{aligned}
$$

It is to be stressed that

$$
Z_{\mathrm{rot}}=\frac{1}{2} \sum_{J=0}^{\infty}(2 J+1) \exp \left(-\frac{J(J+1)}{2 \sigma_{\perp}^{2}}\right) \simeq \sigma_{\perp}^{2}
$$

is a state partition function of type (A6), but our $Z_{\text {coll }}$, derived in Appendix A, is more general, since it is not limited to rotational excitations but is valid for vibrational and transitional spectra as well. Formulas (A12) and (B6) for the spin-dependent level densities cannot be compared directly, because the former uses an intrinsic state density with spherical symmetry and the latter uses an intrinsic state density with axial symmetry.
[1] A. Schiller, L. Bergholt, M. Guttormsen, E. Melby, J. Rekstad, and S. Siem, Nucl. Instrum. Methods Phys. Res. A 447, 498 (2000).

[2] E. Melby, L. Bergholt, M. Guttormsen, M. Hjorth-Jensen, F. Ingebretsen, S. Messelt, J. Rekstad, A. Schiller, S. Siem, and S. W. Ødegård, Phys. Rev. Lett. 83, 3150 (1999).

[3] A. Schiller, A. Bjerve, M. Guttormsen, M. Hjorth-Jensen, F. Ingebretsen, E. Melby, S. Messelt, J. Rekstad, S. Siem, and S. W. Ødegård, Phys. Rev. C 63, 021306(R) (2001).

[4] U. Agvaanluvsan, A. Schiller, J. A. Becker, L. A. Bernstein, P. E. Garrett, M. Guttormsen, G. E. Mitchell, J. Rekstad, S. Siem, A. Voinov, and W. Younes, Phys. Rev. C 70, 054611 (2004).

[5] E. Melby, M. Guttormsen, J. Rekstad, A. Schiller, S. Siem, and A. Voinov, Phys. Rev. C 63, 044309 (2001).

[6] A. Bohr and B. R. Mottelson, Nuclear Structure (Benjamin, Reading, MA, 1975), Vol. II, p. 38.

[7] J. R. Huizenga, A. N. Behkami, R. W. Atcher, J. S. Sventek, H. C. Britt, and H. Freiesleben, Nucl. Phys. A223, 589 (1974).

[8] G. Hansen and A. S. Jensen, Nucl. Phys. A406, 236 (1983).

[9] S. Siem, M. Guttormsen, K. Ingeberg, E. Melby, J. Rekstad, A. Schiller, and A. Voinov, Phys. Rev. C 65, 044318 (2002).

[10] F. Iachello and A. Arima, The Interacting Boson Model (Cambridge University Press, Cambridge, UK, 1987).

[11] J. E. García-Ramos, J. M. Arias, J. Barea, and A. Frank, Phys. Rev. C 68, 024307 (2003).

[12] R. F. Casten and N. V. Zamfir, Phys. Rev. Lett. 87, 052503 (2001).

[13] F. Iachello, Phys. Rev. Lett. 87, 052502 (2001).

[14] D. Kusnezov, Ph.D. thesis, Princeton University, 1988.

[15] D. Kusnezov and F. Iachello, Phys. Lett. B209, 420 (1988).

[16] D. Kusnezov, J. Phys. A 22, 4271 (1989).

[17] D. Kusnezov, J. Phys. A 23, 5673 (1990).

[18] A. Mengoni, A. Ventura, S. Masetti, R. Capote, and D. Kusnezov, J. Nucl. Sci. Technol. Suppl. 2, 766 (2002).

[19] O. Scholten, F. Iachello, and A. Arima, Ann. Phys. (NY) 115, 325 (1978).

[20] D. Kusnezov, Octupole code (unpublished).

[21] F. C. Williams Jr., Nucl. Phys. A133, 33 (1969).

[22] M. Hillman and J. R. Grover, Phys. Rev. 185, 1303 (1969).

[23] R. Capote and R. Pedrosa, Densidad: A code for microscopic calculations of $\mathrm{p}$-h state densities (unpublished), included in the RIPL-2 library (see Ref. [32]).
[24] M. Herman and G. Reffo, Comput. Phys. Commun. 47, 103 (1987).

[25] M. Herman and G. Reffo, Phys. Rev. C 36, 1546 (1987).

[26] M. Herman, G. Reffo, and R. A. Rego, Phys. Rev. C 37, 797 (1988).

[27] N. Cerf, Phys. Rev. C 49, 852 (1994).

[28] N. Metropolis, A. W. Rosenbluth, M. N. Rosenbluth, A. H. Teller, and E. Teller, J. Chem. Phys. 21, 1087 (1953).

[29] E. Mainegra and R. Capote, Comput. Phys. Commun. 150, 43 (2003).

[30] J. R. Huizenga and L. G. Moretto, Annu. Rev. Nucl. Sci. 22, 427 (1972).

[31] F. Iachello and P. Van Isacker, The Interacting Boson-Fermion Model (Cambridge University Press, Cambridge, UK, 1991).

[32] Reference Input Parameter Library, Version 2, http://wwwnds.iaea.org/RIPL-2/.

[33] U. Abbondanno et al. (n_TOF Collaboration), Phys. Rev. Lett. 93, 161103 (2004).

[34] N. Cerf, Phys. Rev. C 50, 836 (1994).

[35] Z. Kargar, A. N. Behkami, and M. N. Nasrabadi, J. Nucl. Sci. Technol. Suppl. 2, 826 (2002).

[36] A. N. Behkami and M. N. Nasrabadi, Commun. Theor. Phys. 37, 457 (2002).

[37] G. Maino, A. Mengoni, and A. Ventura, Phys. Rev. C 42, 988 (1990).

[38] A. Mengoni, A. Ventura, and R. Capote, in Proceedings of the 11th International Symposium on Capture Gamma-Ray Spectroscopy and Related Topics, Pruhonice near Prague, September 2-6, 2002, edited by J. Kvasil, P. Cejnar, and M. Krtička (World Scientific, Singapore, 2003), p. 769.

[39] N. J. Cerf, Phys. Rev. Lett. 76, 2420 (1996).

[40] G. H. Lang, C. W. Johnson, S. E. Koonin, and W. E. Ormand, Phys. Rev. C 48, 1518 (1993).

[41] Y. Alhassid, D. J. Dean, S. E. Koonin, G. Lang, and W. E. Ormand, Phys. Rev. Lett. 72, 613 (1994).

[42] Y. Alhassid, Nucl. Phys. A690, 163c (2001) and references therein.

[43] Y. Alhassid, G. F. Bertsch, and L. Fang, Phys. Rev. C 68, 044322 (2003).

[44] A. S. Iljinov, M. V. Mebel, N. Bianchi, E. De Sanctis, C. Guaraldo, V. Lucherini, V. Muccifora, E. Polli, A. R. Reolon, and P. Rossi, Nucl. Phys. A543, 517 (1992). 